\title{
Intraarterial chemotherapy: a valid option in the treatment of verrucous carcinoma?
}

\author{
Primož Strojan • Alfio Ferlito $\cdot$ Chih-Fung Wu • \\ Alessandra Rinaldo
}

Received: 5 December 2009 / Accepted: 9 December 2009 / Published online: 27 December 2009

(C) Springer-Verlag 2009

More than sixty years have passed since Lauren V. Ackerman first used the term "verrucous carcinoma" in 1948 [1]. This tumor is an unusual and indolent highly differentiated variant of squamous cell carcinoma. Currently, it is referred as a distinct clinicopathologic entity characterized by a favorable prognosis. Verrucous carcinoma is characterized by a high index of clinical suspicion of malignancy, but with a benign histological appearance and no tendency to metastasize neither into the regional lymphatics nor distant sites; it represents a unique diagnostic and therapeutic challenge [2].

Historically, surgery is advocated as the treatment of choice. Operative treatment should not include neck dissection, even though enlarged and tender lymph nodes may be palpated. In fact, histological examination of these lymph nodes has revealed only an inflammatory reaction. Patients receiving initial treatment with surgery enjoyed better survivals than did those treated with irradiation, especially for cases originating in the oral cavity, as suggested by the

This paper was written by members and invitees of the International Head and Neck Scientific Group (http://www.IHNSG.com).

\section{P. Strojan}

Department of Radiation Oncology,

Institute of Oncology, Ljubljana, Slovenia

\section{A. Ferlito $(\bowtie) \cdot$ A. Rinaldo}

Department of Surgical Sciences, ENT Clinic,

University of Udine, Policlinico Universitario,

Piazzale S. Maria della Misericordia, 33100 Udine, Italy

e-mail: a.ferlito@uniud.it

C.-F. Wu

Department of Surgery, Faculty of Medicine,

Kaohsiung Medical University Hospital, Kaohsiung, Taiwan
National Cancer Database records of 2,350 cases of verrucous carcinoma of the head and neck [3].

When deciding between treatment options, a favorable prognosis of verrucous carcinoma dictates two imperatives: the cure of cancer and the preservation of function and/or cosmetic appearance of the treated organ/region. As being local disease, the gold standard of treatment for verrucous carcinoma is surgery assuring high rates of permanent tumor control. However, surgery is limited to the medically fit patients with an operable disease. In locally advanced tumors with locally destructive pattern of growth, often diagnosed in elderly patients, or in tumors extending to neighboring structures more mutilating surgical procedures are necessary or surgery is not an option any more. In such cases, radiotherapy-although being less effective than surgery-is usually considered as a reasonable alternative $[2$, 3]. Recently, the concept of anaplastic transformation after treatment with irradiation, which reasoned many authors into avoiding radiotherapy in the past, was seriously challenged, and the opinion prevailed that it should not affect the decision for this treatment approach. In more recent radiotherapy series, none of the patients with verrucous carcinoma that recurred after radiotherapy had features of anaplastic transformation. It would seem much more probable that the occurrence of a less differentiated lesion after the irradiation of verrucous carcinoma primarily evidences the foci of squamous cell carcinoma that were missed at the initial biopsy [4].

Thus, what therapy might have the potential for preserving the effectiveness of surgery and, at the same time, keeping the organ-sparing capacity of irradiation? One of the strategies introduces chemotherapeutics, either as an upfront modality aimed at reducing the tumor size before surgery or in combination with radiotherapy. However, experience with chemotherapy in verrucous carcinoma is 
scarce, limited to small series and case reports. As in conventional squamous cell carcinomas [5], concomitant chemoradiation was found highly effective and appears to compete favorably with surgery as a preferred treatment modality. According to Strojan et al. [6], after a median follow-up time of 3.4 years, intravenous chemoradiotherapy with vinblastine, methotrexate, and bleomycin resulted in permanent local control in 11 out of 12 patients with verrucous carcinoma, 9 of whom had advanced T3 or T4 tumors. The concomitant administration of chemotherapy seemed to compensate successfully suboptimal effectiveness of irradiation in verrucous carcinoma and allowed safe reduction of irradiation dose below standard 66-70 Gy in the majority of patients. Protracted RTOG grade $\geq 3$ mucositis developed in all patients asking for a temporary treatment interruption in $4 / 10$ patients with the continuous course of irradiation, thereby limiting the number of chemotherapy cycles to 2 in all these patients [6]. The concept of synergistic effect of chemotherapeutics and irradiation in verrucous carcinoma was sporadically confirmed also by others using a wide variety of systemic drugs [7, 8].

Durable tumor control or even cure after chemotherapy alone was also reported. As early as in 1989, following sporadic observations reported in the literature in a case report form only, Auersperg et al. [9] published their experience in a series of 8 cases with verrucous carcinoma (all but 2 sited in the head and neck region) treated primarily with upfront chemotherapy, followed in some of them by surgery (in 4 patients) or irradiation (in 1 patient). After intravenous application of 3- to 4-drug combination chemotherapy (i.e. vinblastine, methotrexate, bleomycin, 5fluorouracil) in all but 2 instances (in 2 patients drugs were infused via intraarterial route), complete response was described in 6 cases. Two out of 3 patients treated solely with multiagent chemotherapy were found free of malignant disease at 2.5 and $8+$ years following the therapy, whereas in the third patient, who was subsequently operated on, no residual disease was found on histopathological examination of the resected specimen. The disease outcome in other 5 patients was as follows: 2 patients (both had surgical resection; 1 of the 2 received also adjuvant chemotherapy) were alive with no evidence of disease at $8+$ and $11+$ years of follow-up; 2 patients experienced disease reappearance that was successfully salvaged with surgery in 1 of them; the patient with cervical verrucous carcinoma died 8 months after the beginning of chemotherapy of respiratory distress with only microscopic residual disease. In this patient, pulmonary fibrosis was found at autopsy. No other major toxic side effects of applied therapy were reported [9].

Recently, an extremely encouraging experience was described by Wu et al. [10] from Kaohsiung Medical University, Taiwan, using intraarterial route for methotrexate application. In a series of 15 patients with oral verrucous carcinoma, the largest described in the literature using chemotherapy as primary treatment modality up to date, $50 \mathrm{mg}$ of methotrexate was infused continuously to the external carotid artery of the lesion side every $24 \mathrm{~h}$ using an implantable port-catheter system inserted under local anesthesia and a portable pump. Infusions were interrupted and switched to weekly intraarterial boluses of $25 \mathrm{mg}$ of methotrexate after white blood cell and platelet counts dropped to $<3,000$ per $\mu \mathrm{L}$ and $10 \times 10^{4}$ per $\mu \mathrm{L}$, respectively. Weekly bolus applications followed until complete disappearance of the gross lesion was recorded. The mean period of continuous methotrexate infusions was 7.5 days and 10 weeks of weekly boluses. One month after treatment discontinuation, complete remission and good cosmetic and functional preservation were obtained in all 15 patients, 8 of whom had locally advanced T3 or T4 lesions. After a mean follow-up time of 42 months (range 24-69 months) they were all still alive with no recurrence of treated tumor. Therefore, the rate of local control was $100 \%$. No catheter-related complications were recorded, whereas bone marrow and mucosal toxicity were mild and tolerable [10]. Favorable results with slightly modified chemotherapy regimen were reported by the same group in a smaller series of 4 patients with penile verrucous carcinoma [11] as well as in a patient with subungual [12] and lower lip verrucous carcinoma [13].

The chemotherapeutics effective in differentiated squamous cell carcinoma were used most frequently in drug combinations or as a single-agent therapy for verrucous carcinoma, i.e. methotrexate, bleomycin, vinblastine, 5fluorouracil and cisplatin. Oral fluoropyrimidines, such as capecitabine and tegafur/uracil, were also prescribed for the patients with verrucous carcinoma, and were variably successful $[7,14,15]$. The choice of chemotherapy schemata was mainly empirical, derived from treating conventional squamous cell carcinoma, although experimental findings were also described and exploited to design most appropriate drug combination $[9,16]$. As shown by the DNA measurements performed during the increased $99 \mathrm{mTc}$-labeled bleomycin accumulation, a relative increase of cells in radiosensitive $G_{2}+M$ compartments and radioresistant $S$ compartment occurred, lasting up to $50 \mathrm{~h}$ after the prolonged administration of vinblastine which was aimed to perturb the cellular kinetics of the tumor. This observation offers a rationale for protracted exposure of cells after vinblastine infusion to the drugs most effective in $G_{2}+M, S$ phases, i.e. bleomycin and methotrexate, and for combining chemotherapy with irradiation $[9,16,17]$.

Continuous drug infusion assures prolonged exposure of tumor cells to the individual agents and, usually, a more favorable toxicity profile, which proved to be of benefit also in verrucous carcinoma when concomitant chemoradiation 
protocol as described by Strojan et al. [6] was used. Theoretically, intraarterial infusion allows higher concentrations of drugs to be delivered to the tumor while minimizing systemic toxicity in comparison with intravenous infusion. The combination of intraarterial route of drug delivery with continuous infusion resulting in protracted and targeted administration of high concentration of anticancer drug to the tumor region was successfully exploited in verrucous carcinoma by Wu et al. [10] as well as by other authors in chemoradiation protocols for conventional squamous cell carcinoma [18].

The results on the use of chemotherapy as the primary therapy of verrucous carcinoma are promising. Chemotherapy used in combination with surgery allows less extensive resections and, consequently, improved functional and cosmetic results. When combined with irradiation, chemotherapy seems to compensate successfully for deficient effectiveness of the former and to allow safe reduction in radiotherapy dose to alleviate its toxicity; at the same time, it preserves the advantage of organ sparing. Both of the above-listed combinations are viable options for patients with inoperable tumors or those that have refused surgery, as well as for patients that are not fit for major surgery or in whom surgery would cause an important functional and/or cosmetic impairment. From this point, the next step forward is obviously the regimen of intraarterial application of methotrexate described by $\mathrm{Wu}$ et al. [10]. Complete response and cure obtained with manageable toxicity in all the treated patients, even though this number is rather low as compared to the series of more usual tumor types, should be a hallmark for all future attempts to improve treatment protocols on verrucous carcinoma.

\section{References}

1. Ackerman LV (1948) Verrucous carcinoma of the oral cavity. Surgery 23:670-678

2. Ferlito A, Rinaldo A, Mannarà GM (1998) Is primary radiotherapy an appropriate option for the treatment of verrucous carcinoma of the head and neck? J Laryngol Otol 112:132-139

3. Koch BB, Trask DK, Hoffman HT, Karnell LH, Robinson RA, Zhen W, Menck HR (2001) National survey of head and neck verrucous carcinoma: patterns of presentation, care, and outcome. Cancer 92:110-120
4. Huang SH, Lockwood G, Irish J, Ringash J, Cummings B, Waldron J, Kim J, Dawson LA, Bayley A, Hope A, O'Sullivan B (2009) Truths and myths about radiotherapy for verrucous carcinoma of larynx. Int J Radiat Oncol Biol Phys 73:1110-1115

5. Pignon JP, le Maître A, Maillard E, Bourhis J, MACH-NC Collaborative Group (2009) Meta-analysis of chemotherapy in head and neck cancer (MACH-NC): an update on 93 randomised trials and 17,346 patients. Radiother Oncol 92:4-14

6. Strojan P, Šoba E, Budihna M, Auersperg M (2005) Radiochemotherapy with vinblastine, methotrexate, and bleomycin in the treatment of verrucous carcinoma of the head and neck. J Surg Oncol 92:278-283

7. Yoshimura Y, Mishima K, Obara S, Nariai Y, Yoshimura H, Mikami T (2001) Treatment modalities for oral verrucous carcinoma and their outcomes: contribution of radiotherapy and chemotherapy. Int J Clin Oncol 6:192-200

8. Tanaka J, Yoshida K, Takahashi M, Suzuki M (1992) A case of verrucous carcinoma of the tongue, effectively treated with preoperative chemotherapy (UFT, CDDP, PEP) and irradiation. Jpn J Cancer Chemother 19:525-527 (in Japanese)

9. Auersperg M, Us-Krašovec M, Lamovec J, Erjavec M, Benulič T, Porenta-Varaspir O (1989) Chemotherapy: a new approach to the treatment of verrucous carcinoma. Radiol Iugos1 23:387-392

10. Wu CF, Chen CM, Shen YS, Huang IY, Chen CH, Chen CY, Shieh TY, Sheen MC (2008) Effective eradication of oral verrucous carcinoma with continuous intraarterial infusion chemotherapy. Head Neck 30:611-617

11. Sheen MC, Sheu HM, Huang CH, Wang YW, Chai CY, Wu CF (2003) Penile verrucous carcinoma successfully treated by intraaortic infusion with methotrexate. Urology 61:1216-1220

12. Sheen MC, Sheen YS, Sheu HM, Wong TW, Lee YY, Wu CF, Wang YW, Chai CY (2005) Subungual verrucous carcinoma of the thumb treated by intra-arterial infusion with methotrexate. Dermatol Surg 31:787-789

13. Sheen MC, Sheu HM, Lai FJ, Lin SD, Wu CF, Wang YW, Lan CCE (2004) A huge verrucous carcinoma of the lower lip treated with intra-arterial infusion of methotrexate. $\mathrm{Br} \mathrm{J}$ Dermatol 151:727-729

14. Salesiotis A, Soong R, Diasio RB, Frost A, Cullen KJ (2003) Capecitabine induces rapid, sustained response in two patients with extensive oral verrucous carcinoma. Clin Cancer Res 9:580 585

15. Kitano H, Kitajima K (1994) Laryngeal verrucous carcinoma: effective treatment with tegafur. Auris Nasus Larynx 21:64-68

16. Auersperg M, Šba E, Vraspir-Porenta O (1977) Intravenous chemotherapy with synchronization in advanced cancer of the oral cavity and oropharynx. Z Krebsforsch 90:149-159

17. Pawlik TM, Keyomarsi K (2004) Role of cell cycle in mediating sensitivity to radiotherapy. Int J Radiat Oncol Biol Phys 59:928942

18. Alkureishi LW, de Bree R, Ross GL (2006) RADPLAT: an alternative to surgery? Oncologist 11:469-480 